\title{
The inter-eye difference in corneal sensitivity in patients with infectious ulcers as measured with the Cochet-Bonnet esthesiometer
}

\author{
Ancy Chakko $\circledast^{1 *}$, Bilto Babu $\circledast^{2 *}$, Satheesh Solomon T. Selvin $\oplus^{3}$ \\ ${ }^{1}$ Lady Willington Hospital, Manali, Himachal Pradesh, India \\ ${ }^{2}$ Shoba Optics, Trissur, Kerala \\ ${ }^{3}$ Department of Ophthalmology, Christian Medical College, Vellore, India \\ ${ }^{*}$ Author was affiliated to the Department of Ophthalmology, Christian Medical College, Vellore, India when the study was done
}

\begin{abstract}
BACKGROUND: The purpose of this study was to quantify the inter-eye difference in corneal sensations in patients with infectious corneal ulcers using the Cochet-Bonnet esthesiometer (CBE).

MATERIAL AND METHODS: Patients with unilateral corneal ulcers of at least $1.5 \mathrm{~mm}$ of the clear cornea in all quadrants meeting the study criteria were identified and enrolled. The clinical features with their etiologies were noted. The corneal sensations were measured with CBE in all four quadrants. Patients were further sub-categorized based on the surface area and the "time to heal" of the ulcers for further correlation and analysis.

RESULTS: This was a cross-sectional study conducted at a tertiary care center. The study included 35 patients, with the majority (30 patients, 85.7\%) diagnosed with fungal etiology. The average corneal sensations in the ulcer and the normal eyes were $52.29 \mathrm{~mm}$ and $57.36 \mathrm{~mm}$, respectively. The inter-eye differences were $3.93 \mathrm{~mm}(\mathrm{p}=0.06)$ and $6.55 \mathrm{~mm}(\mathrm{p}=0.04)$ among patients with an average surface area of $\leq 10 \mathrm{~mm}^{2}$ and $>10 \mathrm{~mm}^{2}$, respectively. Patients who took up to 30 days and $>30$ days to heal had inter-eye differences in a sensation of $0.71 \mathrm{~mm}(\mathrm{p}=0.04)$ and $6.14 \mathrm{~mm}(\mathrm{p}=0.05)$, respectively.

CONCLUSIONS: The mean corneal sensations in all quadrants were less significant in the infectious ulcer eyes than normal eyes. Infectious ulcers with a surface area of $>10 \mathrm{~mm}^{2}$ had greater inter-eye differences in their corneal sensitivities. Patients who took a longer 'time to heal' of $>30$ days showed a significant and greater inter-eye difference in their corneal sensitivities.
\end{abstract}

KEY WORDS: Cochet-Bonnet; corneal sensation; esthesiometer; infectious; ulcer

Ophthalmol J 2021; Vol. 6, 31-37

\section{INTRODUCTION}

The cornea is the most extensively innervated structure of the human body, supplied by the terminal branches of the trigeminal nerve's ophthalmic division as ciliary nerves [1]. These nerves not only carry sensations of pain, touch, or temperature but also regulate the blink reflex, healing after ocular surface injuries and secretion of the tears [2]. In response to any corneal damage, the afferent impulse is triggered when the plexus of corneal nerves is exposed or when sensory neural circuits are disrupted $[2,3]$. Corneal sensitivity is affected significantly by ocular surface disorders or surgeries, which may compromise the protective blink reflex or delay epithelial wound healing. The decrease in sensitivity further can lead to poor wound remodeling and recurrent ocular surface erosions [3-5]. Measurement of the sensitivity is an indirect indicator of 
the cornea's protective mechanisms' integrity and thereby an indirect measure of the corneal nerve function $[4,6]$.

The Cochet-Bonnet esthesiometer (CBE) is traditionally used to measure and quantify the ocular surface sensitivity $[6,7]$. The Cochet-Bonnet esthesiometer employs a non-invasive technique and operates by applying variable pressures on the corneal surface via alteration of the nylon filament's length. As the filament is retracted and made shorter, it becomes stiffer and is easier to feel. Conversely, a longer filament applies less pressure and requires greater corneal sensitivity to detect a touch. Therefore, the corneal sensitivity (the mechano-nociceptor response to the non-invasive mechanical stimulus) of the ocular surface can be measured as the filament's length. Typically, the tip of the nylon filament stimulates nerve terminal endings over a test area of $0.012 \mathrm{~mm}^{2}$ on the cornea, corresponding to around 6-77 terminal endings per square millimeter [3].

The knowledge of alterations in corneal sensitivities in patients with infectious corneal ulcers would be a useful measure in evaluating such patients and understanding the outcome of their healing processes. In our study, we aimed to quantify the corneal sensitivity change in the ulcer eye as compared to the contralateral (normal) eye using the $\mathrm{CBE}$ and assess the inter-eye variation to the size and correlation to the time taken for the ulcers to heal clinically.

\section{MATERIAL AND METHODS}

This was a cross-sectional study conducted at the Department of Ophthalmology of a tertiary-care center in South-India between January and May 2018. The study was approved by the Institutional Review Board and the Ethics Committee (Min. No. 11098, dated 10.01.2018) and followed the Tenets of Declaration of Helsinki. All patients who were diagnosed with infectious corneal ulcers (either admitted for in-patient-care or on follow-up) satisfying the inclusion and exclusion criteria were identified and enrolled after informed consent. This included patients above the age of 18 years with unilateral infectious ulcers with at least $1.5 \mathrm{~mm}$ of the clear cornea in all quadrants. As defined in our study, the corneal ulcer was the presence of a corneal infiltrate and an overlying epithelial defect on clinical examination. Patients with any past history of intra-ocular surgeries or ocular surface procedures in any of the eyes, bilateral corneal ulcers, ocular surface pathologies, past history of contact lens wear, dry eye diseases, use of anesthetic drop in the preceding 6 hours of examination, and mentally-challenged or uncooperative were excluded from the study.

The patients' data were collected, and all patients underwent routine ocular examination as per the clinic protocol following which the corneal sensations were measured with the CBE (Luneau Ophthalmogia, France). Corneal sensitivity measurements were done as per the standard operating protocol by a single investigator. Patients were given a distant target, and keeping the filament perpendicular to the cornea, the sensations were measured around $1.5 \mathrm{~mm}$ inside the limbus. The sensations were assessed at the 12, 3, 6, and 9 o'clock positions in a pre-determined sequential order; superior, temporal, inferior, and nasal, respectively, depending on the eye tested. The procedure was performed to ensure standardization of the order in which the areas were tested. All patients were tested in the clinically uninvolved region (not within the ulcer base) of the cornea. The assessment of each quadrant was started with $60 \mathrm{~mm}$ length of the filament (the lightest touch possible with the instrument). The normal eye was assessed first, followed by the ulcer eye, to avoid the spread of cross-infection. The positive response was considered a blink or when the patient claimed to have felt the touch along with a visible bending of the filament. A negative response was an absent response or an absent blink to the visible bending of the filament. After a negative response, the filament was retracted by $5 \mathrm{~mm}$, and the test repeated until a positive response was obtained. When tested with a $5 \mathrm{~mm}$ length of the filament, a negative response was considered an absent response. The recommended sterilization protocol of the manufacturer was followed before its use on other patients.

All patients underwent corneal scraping for microbiological smear and culture as per the protocol of our hospital. Further information on the clinical details as documented by the treating ophthalmologist was noted from the medical records. The "time to heal" was defined in our study as the number of days taken from the first day of reported symptoms to clinically scar completely and discontinuation of the topical anti-microbial medications.

\section{SAMPLE SIZE CALCULATION}

A pilot study on five patients with the same protocol was done. To detect an inter-eye difference in 
corneal sensitivity of 5 units (using CBE) with an $80 \%$ power at a $5 \%$ significance level, we needed to study 35 eyes in each arm (hypothesis testing of two means) assuming a standard deviation of normal and ulcer eyes as 3 and 9 units respectively. The corneal sensation of each eye was considered as an independent observation for the study sample analysis.

\section{STATISTICAL ANALYSIS}

The data were entered into Microsoft Excel and analyzed using Stata IC v.13.0. Quantitative variables were summarized using mean (SD, standard deviation) for normally distributed variables, median (IQR) for skewed variables. Pearson's correlation coefficient was used to find the correlation of the inter-eye differences of the corneal sensitivities, etiological categorization, surface area, and the "time to heal" of the corneal ulcers. Statistical significance was kept at a $5 \%$ level $(\mathrm{p}<0.05)$.

\section{RESULTS}

A total of 35 patients participated in the study, of which 25 were males (71.4\%). The mean age of the study population was 44.03 years (23-70 years). Etiologically, 30 patients in our study population were diagnosed with fungal ulcers $(85.7 \%)$, two patients each with bacterial (5.7\%) and viral ulcers $(5.7 \%)$, and one with clinically indistinguishable etiology. Seventeen patients had an identifiable microbiological growth - Fusarium (8 patients),
Aspergillus (2 patients), filamentous fungi (5 patients), and one patient each with Curvelaria $S p$ and Exserohilium $S p$.

The mean corneal sensation (average of the four regions measured) in the ulcer and the normal eyes were $52.29 \mathrm{~mm}(\mathrm{SD} \pm 9.59)$ and $57.36 \mathrm{~mm}(\mathrm{SD} \pm 4.01)$, respectively (Fig. 1A). This measured inter-eye difference was statistically significant $(p=0.006)$. Figure $1 b$ represents the inter-eye difference between the sensations of the eyes. The ulcer eyes measured lower than the normal eyes in each of the four quadrants tested. Figure 2 shows the inter-eye mean difference of the measured corneal sensations based on their etiology. Furthermore, patients were categorized based on the ulcers' surface area, viz ulcers with a surface area of $\leq 10$ and $>10 \mathrm{~mm}^{2}$, which had 20 and 15 patients in each group, respectively. The average corneal sensitivities and the significance of the above are shown in Table 1. The patients were followed-up till the complete resolution with no clinical ulcer activity. However, five of our patients were lost to follow up. Table 2 shows the inter-eye differences and the subgroups' statistical significance when categorized based on the "time to heal" $(\mathrm{n}=30)$. The Pearson's correlation of the "time to heal" and the corneal sensations were stronger with an increase in the number of days taken to heal. Pearson's correlation of "time to heal" was $0.373,0.717$, and 0.776 in the three groups, when sub-categorized into $<30,30-60$, and $>60$ days, respectively.
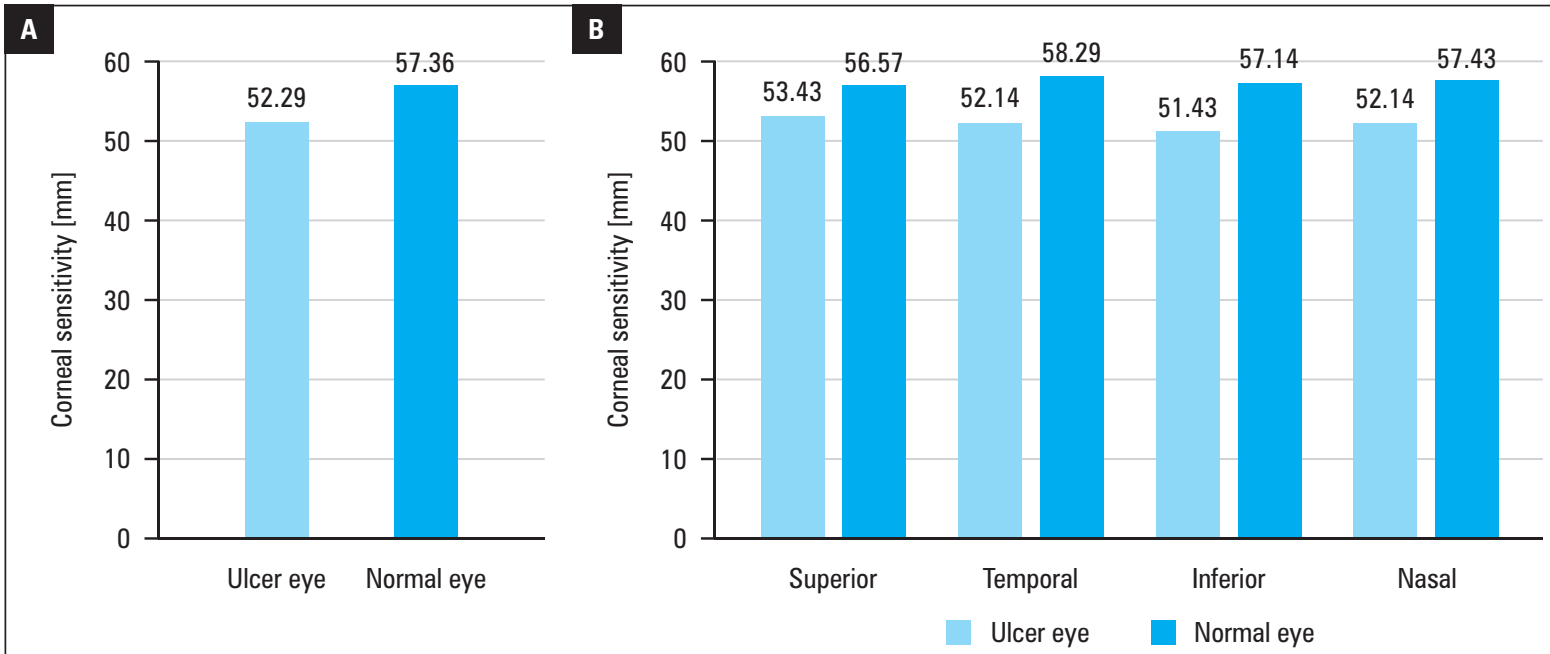

FIGURE 1. A. The average corneal sensitivity of the ulcer and the normal eyes. B. Comparison of the reduction in corneal sensitivity in each quadrant measured using the Cochet-Bonnet esthesiometer in the normal and ulcer eyes 


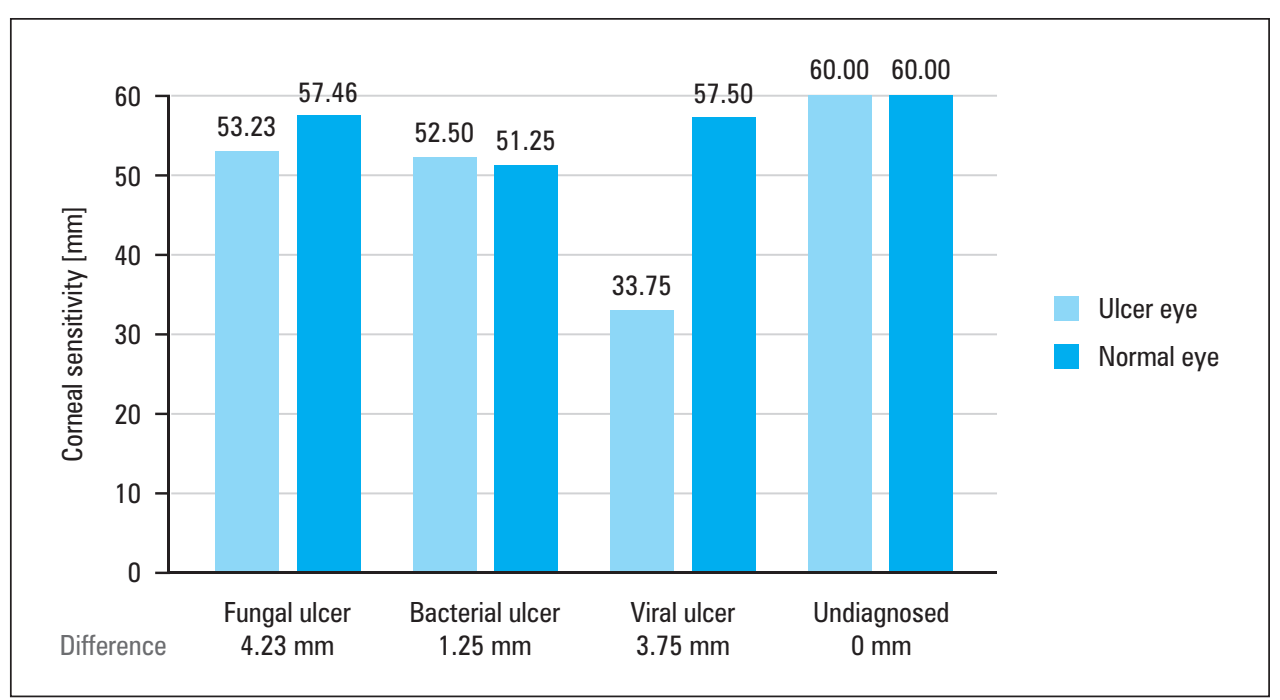

FIGURE 2. The mean corneal sensitivity measured in the normal and the ulcer eyes, in each subgroup categorized based on etiology

Table 1. Mean corneal sensitivity measured with Cochet-Bonnet esthesiometer, categorized based on a surface area of
ulcer
\begin{tabular}{|l|c|c|c|c|}
\hline Size of the ulcer $\left(\mathbf{m m}^{2}\right)$ & No. of patients & $\begin{array}{c}\text { Normal eye } \\
\text { (Mean } \pm \text { SD) }\end{array}$ & $\begin{array}{c}\text { Ulcer eye } \\
\text { (Mean } \pm \text { SD) }\end{array}$ & $\begin{array}{c}\text { Inter-eye difference } \\
\text { (p value) }\end{array}$ \\
\hline$\leq 10$ & 20 & $57.81( \pm 3.39)$ & $53.88( \pm 8.69)$ & $3.93(0.06)$ \\
\hline$>10$ & 15 & $56.75( \pm 4.47)$ & $50.20( \pm 10.6)$ & $6.55(0.04)$ \\
\hline
\end{tabular}

SD - standard deviation

\begin{tabular}{|l|c|c|c|c|}
\hline \multicolumn{3}{|c|}{ Table 2. Mean corneal sensitivity measured with Cochet-Bonnet Esthesiometer, categorized based on "time to heal" } \\
\hline Time to heal (days) & $\begin{array}{c}\text { No. of patients } \\
(\mathbf{n}=\mathbf{3 0})\end{array}$ & $\begin{array}{c}\text { Normal eye } \\
\text { (Mean } \pm \text { SD) }\end{array}$ & $\begin{array}{c}\text { Ulcer eye } \\
\text { (Mean } \pm \text { SD) }\end{array}$ & $\begin{array}{c}\text { Inter-eye difference } \\
\text { (p value) }\end{array}$ \\
\hline$\leq 30$ & 7 & $56.79( \pm 3.13)$ & $56.07( \pm 3.14)$ & $0.71(0.04)$ \\
\hline$>30$ to 60 & 16 & $58.98( \pm 1.60)$ & $53.13( \pm 9.70)$ & $5.86(0.05)$ \\
\hline$>60$ & 7 & $56.96( \pm 3.81)$ & $50.18( \pm 8.34)$ & $6.79(0.04)$ \\
\hline
\end{tabular}

SD - standard deviation

\section{DISCUSSION}

Corneal sensitivity is an indicator of corneal health, and hypoesthesia has an adverse effect that can result in neurotropic conditions, reduced blink rate, and impair epithelial healing. Moreover, a subtle ocular surface injury in such patients can lead to persistent epithelial defect and result in infectious keratitis $[4,5,8]$. Having an intact sensory mechanism in protecting the ocular surface, maintaining blink reflex, and promoting the corneal epithelium's healing response to injury has been established [4, 5]. Alterations in morphology leading to corneal nerve dysfunction following corneal infections, ocular surface surgeries, or other pathologies (e.g., diabetes, dry eye disease, contact lens use, keratoconus, corneal dystrophies, etc.) can have an adverse effect on the ocular sensations $[9,10]$. Trigeminal nerve damage in infected eyes has a direct correlation to the level of inflammation or is directly related to the infectious pathogens causing the dysfunction [9, 10-13]. Exposure to chronic topical medications and long-term self-medication are well-known factors that can result in drug-dependent toxicity affecting corneal sensations $[14,15]$. Thus, the resultant change in the corneal sensitivity is a combination of the factors mentioned above. These potential risk factors form a vicious cycle in the healing of corneal infections.

Most patients enrolled in our study had fungal corneal ulcers $(85.7 \%)$, the spectrum of which cor- 
responds to the general South-Indian data, where clinical fungal ulcers and fungal isolates in scrapings out-number other etiologies, in contrast to developed countries [14-16]. Our study criteria to include only patients with at least $1.5 \mathrm{~mm}$ of the normal (uninvolved) cornea in all quadrants restricted us from including those who had ulcers of eccentric locations or infiltration of the periphery of any particular quadrant. In clinical practice, one would expect to have a larger ulcer and a more pronounced peripheral involvement in bacterial and viral ulcers, respectively. Thus, an etiological distribution, as seen in our study, limited the inclusions of such infectious ulcers.

The average corneal sensations in our study, when compared between the normal and that of the ulcer eyes, showed an inter-eye difference of $5.07 \mathrm{~mm}(\mathrm{p}=0.006)$ with a decreased sensitivity in the latter (Fig. 1A). Further categorization into sub-groups based on etiology also showed a significant inter-eye difference in the sensations in patients with fungal and viral etiologies, the widest difference seen in the latter. The inter-eye difference with the ulcer eyes having lesser sensitivities is possibly due to the inflammation secondary to the microbiological infectious etiology causing damage to the plexus of nerves and the terminal receptors. Though in our study population, only two patients had viral keratitis, the average mean sensitivity between both the eyes showed a clinical and a statistical difference of $23.75 \mathrm{~mm}(\mathrm{p}<0.05)$ (Fig. 2). The study by Fernandez et al. [10] on patients with viral keratitis too showed a mean reduction in the ulcer eyes $(16.27 \mathrm{~mm})$ as compared to the contralateral eye $(52.19 \mathrm{~mm})$. Studies have shown that active herpetic keratitis presents with relative hypoesthesia, and this drop in the sensation can persist beyond the active period, especially when patients have recurrent episodes. This is logical considering the virus resides in the ganglion, in its dormant form [11]. These are not just true with viral etiology alone but have also been reported in cases of bacterial and fungal keratitis $[10,13,17]$. In such cases of microbial ulcers, inflammation can be a resultant of injury to the corneal nerve plexus leading to impaired corneal sensitivity. However, the sensations in patients with bacterial keratitis in our study were measured higher, which could be secondary to corneal hypersensitivity in the acute inflammatory phase. However, this finding cannot be extrapolated as a rule to all patients with bacterial ulcers. This could also be due to the fact that following nerve injury, the injured corneal nerves themselves exhibit altered functional properties and paradoxically display abnormal intrinsic electrical excitability leading to hyperresponsiveness [13]. Only two patients with bacterial ulcers in our study fitting the inclusion criteria indicates the general pattern of virulence of bacteria progressing faster to become larger ulcers. The rate of spread to involve a large part of the cornea could also be the reason why such patients did not fit our study criteria (to have at least $1.5 \mathrm{~mm}$ of the clear cornea in all quadrants). Also, patients presenting to the tertiary-care-center would be the ones who did not respond to the first-line treatment given by their primary eye care physicians and presented late, whereas patients with small ulcers who would have otherwise fit into the study criteria responded to the empirical treatment and healed favorably. The ulcers leading to the eyes having decreased corneal sensitivities and the eyes which take a longer time to heal may have an inherent poor sensation, both of which are inter-related and theoretically possible. However, the inter-eye difference with decreased sensitivities in the ulcer eyes, as shown in our study, favors the infections that have led to decreased corneal sensations. Furthermore, the comparable mean values of the corneal sensations of the normal eyes in our study to the one by Kotak et al. [18] in a similar population suggests that the decreased corneal sensations are likely to be secondary to the infectious ulcers and not vice versa. One needs to remember that the corneal ulcers leading to decreased sensations, further susceptibility to develop corneal infections, and the delay in ulcer healing could again form a vicious cycle in these patients.

Categorization of the patients to the ulcer's size (surface area $\leq 10 \mathrm{~mm}^{2}$ and $>10 \mathrm{~mm}^{2}$ ) showed a higher inter-eye difference in the corneal sensations in the group with the larger surface area. Patients with ulcer's area $\leq 10 \mathrm{~mm}^{2}$ (20 patients) showed a mean difference of $3.93 \mathrm{~mm}$ between the ulcer and the normal eyes, which was close to being statistically significant $(\mathrm{p}=0.06)$. The inter-eye difference was higher $(6.55 \mathrm{~mm}, \mathrm{p}=0.04)$ with a better statistical significance in patients with ulcer's area $>10 \mathrm{~mm}^{2}$ (15 patients) (Tab. 1). On further sub-analyzing, the group of patients with ulcer's area $\geq 20 \mathrm{~mm}^{2}$, the inter-eye difference was $10.21 \mathrm{~mm}$, which was clinically and statistically more significant. Measurement of surface area as a protocol to determine an outcome measure is not feasible in all clinical scenarios. Having the diameter measured in a clinical situation is more logi- 
cal. On back calculating, the patients with ulcer's area $>10 \mathrm{~mm}^{2}$ would have an average diameter of $3.6 \mathrm{~mm}$, which can be used as a guideline during clinical assessment. The subset of patients accordingly took a longer time to heal (average 76.1 days). Similarly, the inter-eye difference in the sensations doubled amongst the group of patients with corneal ulcers $>5 \mathrm{~mm}$ in diameter $(\mathrm{p}<0.05)$. The inflammation level is directly proportional to the nerve receptors' damage by the infectious pathogens in the infected eyes, which is evident from our study, where the larger the ulcer, the more significant the drop in the corneal sensations. When the tested corneal quadrants were studied individually for their respective corneal sensitivity values, all the four quadrants had a significant inter-eye difference in the values (Fig. 1B). The temporal quadrant had the maximum inter-eye difference followed by inferior, nasal, and superior regions, respectively. The inherent property of the superior region of the cornea to have the least sensation as compared to the other regions [7] could be the reason for the region having the least drop and thereby a smaller inter-eye difference among the quadrants.

"Time to heal", as defined in our study, was the time taken for the ulcer to heal to the point where the use of anti-microbial drops could be stopped, starting from the first day of appearance of symptoms. Patients were categorized based on the same as those who took up to 30 days, 30 to 60 days, and $>60$ days to heal (Tab. 2). The Pearson's correlation followed a linear pattern and became stronger as the number of days to heal increased. When the patients who took $>30$ days to recover were categorized into a single group, the measured inter-eye difference was $6.14 \mathrm{~mm}(\mathrm{p}=0.04)$, which was statistically significant with a clinically measurable difference. Assessing the corneal sensation in corneal pathologies with no predisposing risk factors should be considered essential, as absent or decrease corneal sensation may be a sinister sign. It is critical and important to test the unaffected area for the corneal sensitivity measurement and compared to the same in the contralateral (normal) eye. These will aid in the follow-up and act as a surrogate clinical test for the "time to heal" in infectious ulcers when done meticulously.

In most clinical situations, when patients present late, a combination of different medications would have been trailed. In our study too, about $94.28 \%$ of the patients were on combinations of the treatment protocol and supplementary drugs for a vari- able period of time at the moment of enrollment and corneal sensitivity measurement. These confounding factors and the combinations were too many to derive useful comparisons between drug regimens. As our study required at least $1.5 \mathrm{~mm}$ rim of the uninvolved cornea, patients with large or eccentrically located ulcers were excluded, which are the more pertinent in understanding their clinical healing outcome. However, developing a standardized protocol to measure the sensations in such patients may not be uniform to extrapolate to a larger population to understand the outcome of the disease. Our study's other limitations included patients studied at different stages of the ulcer: its healing process could have had a hyper- or hyposensitive response, which could not be completely eliminated. Also, having fewer patients with bacterial and viral ulcers made the cross-comparison between etiologies not possible.

\section{CONCLUSION}

A definitive and statistically significant inter-eye difference is seen in the corneal sensations, with infectious ulcers eyes having decreased measured values. The reduction in corneal sensitivity measured with CBE was statistically more significant in eyes with infectious ulcers with surface area $>10 \mathrm{~mm}^{2}$ and amongst eyes that took $>30$ days to heal.

\section{Statement of competing interests}

The authors have not conflicts of interests to disclose.

\section{Financial support and funding}

None.

\section{Acknowledgement}

Nil.

\section{REFERENCES}

1. Cruzat A, Pavan-Langston D, Hamrah P. In vivo confocal microscopy of corneal nerves: analysis and clinical correlation. Semin Ophthalmol. 2010; 25(5-6): 171-177, doi: 10.3109/08820538.2010.518133, indexed in Pubmed: 21090996.

2. Shaheen BS, Bakir M, Jain S. Corneal nerves in health and disease. Surv Ophthalmol. 2014; 59(3): 263-285, doi: 10.1016/j.survophthal.2013.09.002, indexed in Pubmed: 24461367.

3. Bragheeth MA, Dua HS. Corneal sensation after myopic and hyperopic LASIK: clinical and confocal microscopic study. Br J Ophthalmol. 2005; 89(5): 580-585, doi: 10.1136/bjo.2004.046888, indexed in Pubmed: 15834089.

4. Brennan NA, Bruce AS. Esthesiometry as an indicator of corneal health. Optom Vis Sci. 1991; 68(9): 699-702, doi: 10.1097/00006324199109000-00004, indexed in Pubmed: 1745494. 
5. Collins M, Seeto R, Campbell L, et al. Blinking and corneal sensitivity. Acta Ophthalmol (Copenh). 1989; 67(5): 525-531, doi: 10.1111/ j.1755-3768.1989.tb04103.x, indexed in Pubmed: 2589051.

6. Booranapong W, Tanthuvanit P, Suwannik A. Corneal sensation in the normal Thai population. Siraj Medical J. 2005; 57: 262-65.

7. Chao C, Stapleton F, Badarudin E, et al. Ocular surface sensitivity repeatability with Cochet-Bonnet esthesiometer. Optom Vis Sci. 2015; 92(2): 183-189, doi: 10.1097/0PX.0000000000000472, indexed in Pubmed: 25546826.

8. Müller LJ, Marfurt CF, Kruse F, et al. Corneal nerves: structure, contents and function. Exp Eye Res. 2003; 76(5): 521-542, doi: 10.1016/s00144835(03)00050-2, indexed in Pubmed: 12697417.

9. Cruzat A, Schrems WA, Schrems-Hoesl LM, et al. Contralateral Clinically Unaffected Eyes of Patients With Unilateral Infectious Keratitis Demonstrate a Sympathetic Immune Response. Invest Ophthalmol Vis Sci. 2015; 56(11): 6612-6620, doi: 10.1167/iovs.15-16560, indexed in Pubmed: 26465889.

10. Marcos-Fernández M, Tabernero S, Herreras J, et al. Impact of herpetic stromal immune keratitis in corneal biomechanics and innervation. Graefe's Arch Clin Experiment Ophthalmol. 2017; 256(1): 155-161, doi: 10.1007/s00417-017-3826-3.

11. Rolinski J, Hus I. Immunological aspects of acute and recurrent herpes simplex keratitis. J Immunol Res. 2014; 2014: 513560, doi: 10.1155/2014/513560, indexed in Pubmed: 25276842.

12. Cruzat $A$, Witkin $D$, Baniasadi $N$, et al. Inflammation and the nervous system: the connection in the cornea in patients with infectious kerati- tis. Invest Ophthalmol Vis Sci. 2011; 52(8): 5136-5143, doi: 10.1167/ iovs. 10-7048, indexed in Pubmed: 21460259.

13. Belmonte C, Acosta MC, Gallar J. Neural basis of sensation in intact and injured corneas. Exp Eye Res. 2004; 78(3): 513525, doi: 10.1016/j.exer.2003.09.023, indexed in Pubmed: 15106930

14. Bharathi MJ, Ramakrishnan R, Meenakshi R, et al. Microbial keratitis in South India: influence of risk factors, climate, and geographical variation. Ophthalmic Epidemiol. 2007; 14(2): 61-69, doi: 10.1080/09286580601001347, indexed in Pubmed: 17464852.

15. Ravinder K, Madhav MV. Archa $\neg$ na J, Pandurang J. Clinical evaluation of corneal ulcer among patients attending teaching hospital. Int J Contem Med Res. 2016; 3(4): 949-952.

16. Lin CC, Lalitha P, Srinivasan M, et al. Seasonal trends of microbial keratitis in South India. Cornea. 2012; 31(10): 1123-1127, doi: 10.1097/ IC0.0b013e31825694d3, indexed in Pubmed: 22868629.

17. Tesón M, Calonge M, Fernández I, et al. Characterization by Belmonte's gas esthesiometer of mechanical, chemical, and thermal corneal sensitivity thresholds in a normal population. Invest Ophthalmol Vis Sci. 2012; 53(6): 3154-3160, doi: 10.1167/iovs.11-9304, indexed in Pubmed: 22511623.

18. Selvin S, Kotak S, Christina M. Establishing the normal range of corneal sensitivity in an Indian Population using the CochetBonnet esthesiometer. Curr Med Issu. 2020; 18(3): 170, doi: 10.4103/cmi.cmi_24_20. 